\title{
Circumferential Resection Margin
}

National Cancer Institute

\section{Source}

National Cancer Institute. Circumferential Resection Margin. NCI Thesaurus. Code C46085.

The non-peritonealised bare area of rectum, comprising anterior and posterior segments, when submitted as a surgical specimen resulting from excision of cancer of the rectum. 